\title{
Breast DCE-MRI: lesion classification using dynamic and morphological features by means of a multiple classifier system
}

\author{
Roberta Fusco ${ }^{1}$, Massimiliano Di Marzo ${ }^{2}$, Carlo Sansone ${ }^{3}$, Mario Sansone ${ }^{3}$ and Antonella Petrillo ${ }^{1 *}$
}

\begin{abstract}
Background: In breast magnetic resonance imaging (MRI) analysis for lesion detection and classification, radiologists agree that both morphological and dynamic features are important to differentiate benign from malignant lesions. We propose a multiple classifier system (MCS) to classify breast lesions on dynamic contrast-enhanced MRI (DCE-MRI) combining morphological features and dynamic information.

Methods: The proposed MCS combines the results of two classifiers trained with dynamic and morphological features separately. Twenty-six malignant and 22 benign breast lesions, histologically proven, were analysed. The lesions were subdivided into two groups: training set (14 benign and 18 malignant) and testing set (8 benign and 8 malignant). Volumes of interest were extracted both manually and automatically. We initially considered a feature set including 54 morphological features and 98 dynamic features. These were reduced by means of a selection procedure to delete redundant parameters. The performance of each of the two classifiers and of the overall MCS was compared with pathological classification.
\end{abstract}

Results: We obtained an accuracy of $91.7 \%$ on the testing set using automatic segmentation and combining the best classifier for morphological features (decision tree) and for dynamic information (Bayesian classifier). With implementation of the MCS, an increase in accuracy of $12.5 \%$ and of $31.3 \%$ was obtained compared with the accuracy of the Bayesian classifier tested with dynamic features and with that of the decision tree tested with morphological parameters, respectively.

Conclusions: An MCS can optimise the accuracy for breast lesion classification combining morphological features and dynamic information.

Keywords: Breast cancer, Dynamic contrast-enhanced MRI, Multiple classifier system, Morphological features, Decision tree, Dynamic features, Bayesian classifier

\section{Key points}

- An MCS combined two classifiers trained with morphological and dynamic features.

- A decision tree was used for classifying morphological features.

- A Bayesian classifier was used for classifying dynamic features.

\footnotetext{
* Correspondence: a.petrillo@istitutotumori.na.it

'Department of Diagnostic Imaging, Radiant and Metabolic Therapy, "Istituto Nazionale Tumori Fondazione Giovanni Pascale—IRCCS", Via Mariano

Semmola, 80131 Naples, Italy

Full list of author information is available at the end of the article
}

- Combining morphologic and dynamic features, $92 \%$ accuracy can be obtained.

\section{Introduction}

Breast cancer is the most common cancer among women in the western world. To date it is the second leading cause of cancer death in women (after lung cancer) and is estimated to cause $15 \%$ of cancer deaths [1]. Therefore, screening for early diagnosis of breast cancer is of great interest.

The currently most widespread screening method is $\mathrm{X}$-ray mammography [2]. However, this method is not adequate for young women in the presence of dense 
breasts. Moreover, detection and characterisation of breast lesions on mammography is difficult because of the lack of functional information.

Dynamic contrast-enhanced magnetic resonance imaging (DCE-MRI) has demonstrated a great potential for screening high-risk women, staging newly diagnosed breast cancers, and assessing therapy effects [1], thanks to the possibility of visualising three-dimensional (3D) high-resolution dynamic (functional) information, not available with mammography or with ultrasound. Therefore DCE-MRI is gaining popularity as an important tool for breast cancer diagnosis [3].

For breast lesion detection and classification using DCE-MRI, radiologists agree that both morphological and dynamic features are important [4-6]. On the one hand, morphological features aim to quantify lesion characteristics well assessed in the breast MRI lexicon [6]: round shape and smooth margin for benign lesions; irregular shape and margins for malignant lesions. On the other hand, dynamic information has shown great potential for quantifying tumour vascularity [6-8]: malignant lesions usually show early enhancement with rapid washout, whereas benign lesions typically show a slow increase followed by persistent enhancement [6].

Recent studies have attempted to take advantage of morphological features and dynamic information: dynamic and morphological data, generally separately, have been used both for segmentation of volume of interests (VOIs) [7-11] and for lesion classification [6-15]. Nie et al. [4] demonstrated that quantitative analysis of the morphology and texture features of breast lesions is feasible. These features could be selected by an artificial neural network for differential diagnosis between breast cancer and benign breast lesions. Agner et al. [14] showed that good performances could be yielded using a probabilistic boosting tree classifier in conjunction with textural kinetic features. However, when the dataset included both textural kinetic and morphologic features, the performance was lower. Zheng et al. $[13,16]$ investigated the use of a feature set including dynamic, spatial, and morphological features with a linear classifier.

To the best of our knowledge, a multiple classifier system (MCS) for classification of breast lesions using morphological and dynamic features in DCE-MRI has not yet been presented, although the idea of combining multiple classifiers is not new. For example, Keyvanfard et al. [17] proposed an MCS composed of three classifiers that used dynamic features to classify breast lesions in DCE-MRI, but morphological features were not used in their study.

Our aim was to propose an MCS for classifying breast lesions using both morphological and dynamic features on DCE-MRI. As classifiers, we used those best suited for the problem at hand, according to our previous study [18].

\section{Materials and methods}

\section{Patient selection}

Forty-eight women (age $51 \pm 18$ years, mean \pm standard deviation) with pathologically proven benign or malignant lesions were examined retrospectively. All of these subjects had undergone DCE-MRI at our cancer centre. Twenty-six lesions were malignant and 22 were benign. The lesions were subdivided into two groups (Table 1): training set (14 benign and 18 malignant) and testing set ( 8 benign and 8 malignant). All patients provided informed consent for the use of their data for research purposes. This retrospective study was performed according to regulations issued by our local Institutional Review Board.

\section{Data acquisition}

The patients underwent imaging with a 1.5-T scanner (Magnetom Symphony; Siemens Medical System, Erlangen, Germany) equipped with a dedicated breast coil with 16 channels. Turbo spin-echo T2-weighted axial images (TR/TE 4000/56 ms; flip angle $180^{\circ}$; field of view $340 \times$ $340 \mathrm{~mm}^{2}$; matrix $385 \times 385$; slice thickness $2 \mathrm{~mm}$; no interslice gap; 56 slices covering the entire breast volume) and turbo spin-echo T1-weighted fat-saturated axial images (TR/TE 564/12 ms; flip angle $90^{\circ}$; field of view $350 \times 350 \mathrm{~mm}^{2}$; matrix $512 \times 256$; slice thickness $2 \mathrm{~mm}$; no interslice gap; 80 slices covering the entire breast volume) were acquired for morphological imaging. T1-weighted fast low-angle shot 3D coronal images were acquired (TR/TE 9.8/4.76 ms; flip angle $25^{\circ}$; field of view $330 \times 247 \mathrm{~mm}^{2}$; matrix $256 \times 128$; partition thickness $2 \mathrm{~mm}$; no interslice gap; acquisition time $56 \mathrm{~s} ; 80$ slices covering the entire breast volume). One series was acquired before and nine series after intravenous injection of

Table 1 Pathology of lesions included in the training set and in the testing set

\begin{tabular}{lll}
\hline Pathology & Training set & Testing set \\
\hline Malignant & 7 & 4 \\
Invasive ductal & 2 & 1 \\
Invasive lobular & 5 & 2 \\
Invasive ductal lobular & 4 & 1 \\
Ductal carcinoma in situ & 18 & 8 \\
Subtotal & & \\
Benign & 10 & 5 \\
Fibroadenoma & 2 & 1 \\
Ductal hyperplasia & 1 & 1 \\
Fibrocystic dysplasia & 1 & 1 \\
Intraductal papilloma & 14 & 8 \\
Subtotal & 32 & 16 \\
Grand total &
\end{tabular}


$0.1 \mathrm{mmol} / \mathrm{kg}$ body weight of a positive paramagnetic contrast material (Gd-DOTA; Dotarem, Guerbet, Roissy CdG Cedex, France). An automatic injection system was used (Spectris Solaris EP MR; MEDRAD, Inc, Indianola, PA, USA) and the injection flow rate was set to $2 \mathrm{ml} / \mathrm{s}$ followed by a flush of $10 \mathrm{ml}$ saline solution at the same rate.

\section{Manual segmentation}

Regions of interest (ROIs) to cover the entire tumour volume were drawn manually slice by slice, by a radiologist with 22 years of experience in breast MRI, on T1weighted images obtained by subtracting the unenhanced image from the fifth contrast-enhanced image using the turbo spin-echo T2-weighted images as a guide. For each patient, all of the slices including the lesion were used. The segmentation was performed with OsiriX v.3.8.1 (Fig. 1a).

\section{Automatic segmentation}

The automatic segmentation algorithm included three steps. The first involves a breast mask extraction by means of an automatic intensity threshold estimation (Otsu thresholding) on the parametric map obtained, considering the sum of intensity differences (SOD) calculated voxel by voxel. This parameter describes the dynamic information of the whole curve and reflects the history of contrast agent enhancement with time:

$$
S O D_{p}=\operatorname{Pre}_{p}+\sum_{i=1}^{T} \mid \operatorname{Post}_{p}(i)-\text { Post }_{p}(i-1) \mid
$$

where $S O D_{p}$ is the SOD for the $p$ voxel, $P r e_{p}$ is the precontrast intensity, $\operatorname{Post}_{p}(i)$ is $i$ th post-contrast scan and $T$ is the total number of scans.

The second step included hole-filling and leakage removal by means of morphological operators: closing is required to fill the holes on the boundaries of breast mask; filling is required to fill the holes within the breasts; and erosion is required to reduce the dilation obtained by the closing operation $[19,20]$.
The third step included the extraction of suspicious ROIs. The dynamic features of each voxel were analysed. A voxel was assigned to a suspicious ROI if it satisfies two conditions: the maximum of its normalised timeintensity curve should be greater than 0.3 , and the maximum signal intensity should be reached before the end of the scan time. The first condition assures that the voxels within the ROI have a significant contrast agent uptake (thus excluding slow enhancement curves) and the second condition is required for the time-intensity pattern with plateau or wash-out $[19,20]$. The choice of the 0.3 threshold was based on the findings by Torricelli et al. [21]. In their study, lesions with an enhancement lower than 50\% above the baseline were considered nontumoral. They also noticed that lowering the threshold to $40 \%$ improved the accuracy of diagnosis. We proposed a threshold of $30 \%$ in order to reduce the number of false negatives. All procedures were implemented in Matlab R2008a using Image toolbox (Fig. 1b).

\section{Morphological and dynamic features}

According to our previous studies [18-20], we considered a feature set including 54 morphological features and 98 dynamic features. The main categories of morphological features included both two-dimensional (2D) and 3D parameters. The 2D morphological features were: mean, standard deviation, maximum and minimum value of areas; perimeters; complexity; radial length; and spiculation. These were obtained slice by slice and then median values were calculated. The 3D features were circularity, compactness, smoothness, roughness, sphericity, eccentricity, volume, rectangularity, solidity, convexity, curvature, and edge $[4,5,7]$. These were obtained on the entire segmented 3D volume. For dynamic features the main categories included area, maximum intensity ratio, relative enhancement, relative enhancement slope, basal signal, perfusion index, SOD, wash-in, wash-out, and time to peak $[3,7,11,13]$.

The number of morphological and dynamic features was reduced by a feature selection procedure to remove uninformative and unimportant morphological features.

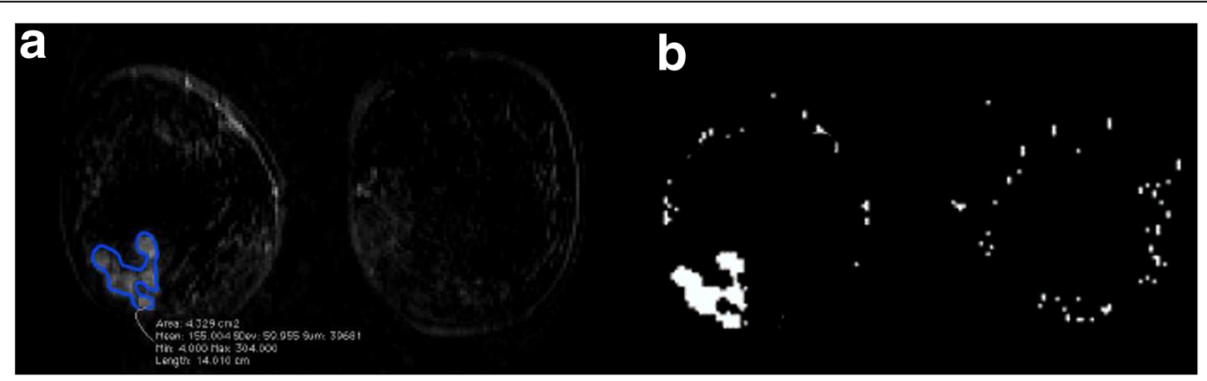

Fig. 1 Example of ROI manual segmentation (a) and ROI automatic segmentation (b) for the same multifocal confluent lesion at the external lower quadrant of the right breast 
To keep the loss of information to a minimum we tested the correlation-based feature selection method and the consistency feature selection method with several searches: the forward search, the backward search, the bidirectional search, the greedy search, and feature ranking methods. The morphological features obtained by the selection procedure were area, eccentricity, compactness, and perimeter. The dynamic features retained by the selection procedure were the sum of intensity difference, basal signal, and relative enhancement slope. Table 2 presents the mathematical formula for both morphological and dynamic features retained by the selection procedure which were used to train and test the classifiers.

The classifiers were trained with the morphological and dynamic features extracted by both manual and automatic segmentation to assess whether the automatic procedure could affect the MCS performance.

\section{VOI classification}

The proposed MCS combines the results of two classifiers trained separately with dynamic and morphological features, respectively (Fig. 2). In particular, the weighted sum of probability of malignancy and the probability of benignancy of the two chosen classifiers as proposed by De Santo et al. [22] were considered.

The choice of the proposed classifier was based on a previous study [18], where a decision tree (collaborative filtering $=0.25$; unpruned $=$ true) and a Bayesian classifier $($ Kernel estimator $=$ false) gave $u$ s the best results when trained on morphological and dynamic features, respectively. We estimated the classifier parameters using the training data and the subset of features obtained by feature selection methods varying classifier parameter values and evaluating the accuracy. A 10-fold crossvalidation procedure was chosen to train the classifiers.

Then, in order to combine the results of the two classifiers, each suspicious voxel within the VOI was first classified as benign or malignant based on dynamic information. The whole VOI was then classified as malignant if the number of malignant voxels $\left(n_{\mathrm{m}}\right)$ within the VOI was higher than benign voxels $\left(n_{\mathrm{b}}\right)$ within the same VOI.

The probability of malignant lesions $\left(D_{\mathrm{m}}\right)$ and the probability of benign lesions $\left(D_{\mathrm{b}}\right)$ were calculated as follows:

$$
\begin{aligned}
& D_{\mathrm{m}}=n_{\mathrm{m}} / N \\
& D_{\mathrm{b}}=n_{\mathrm{b}} / N
\end{aligned}
$$

where $N$ is the total number of voxels in the lesion.

Morphological features were instead calculated for the whole VOI and used to classify the lesion into malignant and benign. In this case, the probability of malignancy and the probability of benignancy were $M_{\mathrm{m}}$ and $M_{\mathrm{b}}$, respectively.

Finally, the VOI was classified as malignant if $\alpha D_{\mathrm{m}}$ $+\beta M_{\mathrm{m}}>\alpha D_{\mathrm{b}}+\beta M_{\mathrm{b}}$, where $\alpha$ and $\beta$ were multiplicative coefficients $(\alpha+\beta=1)$ which must be suitably chosen in order to maximise the accuracy (Fig. 2).

We estimated $\alpha$ and $\beta$ coefficients using the test set lesions group, the subset of features retained by feature selection methods, and the optimised classifiers obtained by training data set analysis and evaluating the accuracy of the MCS. A leave-one-out cross-validation procedure was chosen as suggested by Torricelli et al. [21].

Machine learning analysis was performed using Weka open source software (http://www.cs.waikato.ac.nz/ml/ weka/).

\section{Statistical analysis}

Sensitivity, specificity, positive and negative predictive values, and accuracy were reported for each of the two classifiers and for the MCS in the case of both the manual and the automatic segmentation procedure. The McNemar test was used to assess differences between manual and automatic segmentation. Calculations were

\begin{tabular}{|c|c|c|}
\hline \multicolumn{2}{|l|}{ Feature } & \multirow{2}{*}{$\begin{array}{l}\text { Formula } \\
n_{k} d x d y \text { where } n_{k} \text { is number of voxels in the } k \text { th slice of the } \mathrm{ROI}, d_{x} \text { and } d_{y} \text { represent size of voxels }\end{array}$} \\
\hline Morphological & Area, $A_{k}$ & \\
\hline & Perimeter length, $P_{k}$ & $b_{k}$ where $b_{k}$ is number of boundary voxels in the $k$ th slice of the $\mathrm{ROI}$ \\
\hline & Compactness in $3 \mathrm{D}, \mathrm{COMP}$ & $\begin{array}{l}\frac{S^{2}}{V_{30}} \text { where } S \text { is the surface and } V \text { is the volume, defined as follows: } s=\sum_{x} \sum_{y} \sum_{z} b_{R O I}(x, y, z) v_{\text {size }} \text { slice } e_{t h} \\
\text { where } v_{\text {size }} \text { is the voxel size, slice } \text { th }_{\text {th }} \text { is the slice thickness; and } V=n_{R O} d x d y d z \text { where } n_{R O I} \text { is total number } \\
\text { of voxels in the ROI }\end{array}$ \\
\hline & Eccentricity, ECC & $\frac{\sqrt{a^{2}-b^{2}}}{a}$ where $a$ is the major axle shaft and $b$ is the lower one \\
\hline \multirow[t]{3}{*}{ Dynamic } & Basal signal, BS & Signal intensity before contrast injection \\
\hline & Relative enhancement, $R E\left(t_{i}\right)$ & $\frac{S\left(t_{i}\right)-B S}{B S}$ where $t_{i}$ is the ith temporal instant \\
\hline & Sum of local differences, SOD & $\mathrm{SOD}_{p}=\operatorname{Pre}_{p}+\sum \mid$ Post $_{p}(i)-$ Post $_{p}(i-1) \mid$ \\
\hline
\end{tabular}

Table 2 Performance on the testing set obtained by the single classifier 


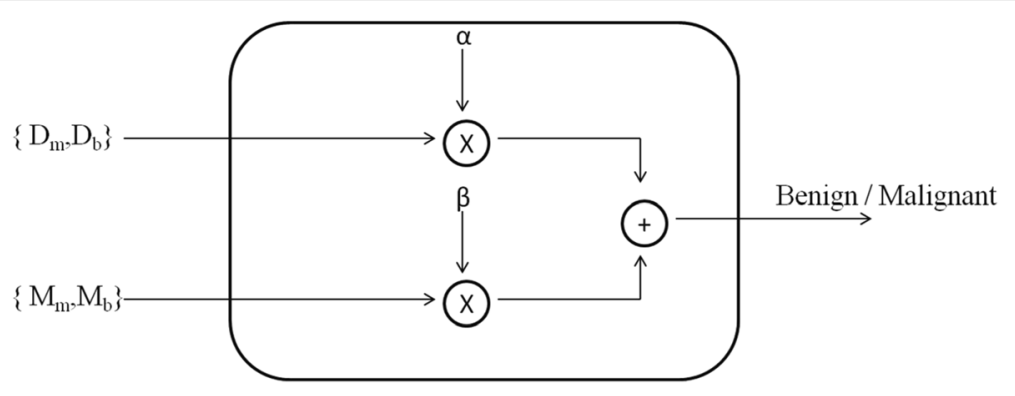

Fig. 2 Illustration of the MCS as the combination of a classifier tested with morphological features and a classifier tested with dynamic information. $D_{\mathrm{m}}$ probability of malignant lesions, $D_{\mathrm{b}}$ probability of benign lesions, $M_{\mathrm{m}}$ probability of malignity, $M_{\mathrm{b}}$ probability of benignity, $a$ and $\beta$ multiplicative coefficients $(\alpha+\beta=1)$

performed using the Statistic Toolbox of Matlab R2008a. Values of $p$ lower than $<0.05$ was considered significant.

\section{Results}

Table 2 presents the morphological and dynamic features retained by the feature selection procedure and that were used to train the individual classifier. Table 3 presents the results obtained on the testing set by each of the two classifiers using dynamic or morphological features, both for manual and automatic segmentation. We observed a significant difference between the performances of classifiers when manual or automatic segmentation was performed.

As far as the choice of optimal values for $\alpha$ and $\beta$ is concerned, we report in Fig. 3 the percentage of correctly classified lesions versus $\alpha$. The best compromise among sensitivity (92.3\%), specificity (90.9\%), positive predictive value (92.3\%), negative predictive value (90.9\%), and overall accuracy (91.7\%) resulted in $\alpha=0.75$ with only two false negatives and two false positives. With MCS implementation, an increase in accuracy of $12.5 \%$ and of $31.3 \%$ was obtained when comparing the Bayesian classifier using dynamic features with the decision tree using morphological features, respectively.

\section{Discussion}

The aim of this study was to propose an MCS to classify breast lesions on DCE-MRI. The proposed MCS combines the results of two classifiers trained and tested using dynamic and morphological features separately.

It is well known that training machine learning classifiers with a large number of features can lead to classifier overfitting, reducing the generalisation capabilities of the classifiers and slowing down the training process. As a consequence, a selection procedure was performed. The features retained by the selection procedure (area, eccentricity, compactness, and perimeter as morphological features; and SOD, basal signal, and relative enhancement slope as dynamic features) were used to assess the performance of the single classifier and of the MCS.

In our previous studies $[18,20]$ we analysed the performance of several classifiers (multilayer perceptron, support vector machine, Bayes classifier decision tree), in conjunction with dynamic and morphological features, separately using a ROI manual selection slice by slice. In this study we compared the results of accuracy to differentiate benign from malignant lesions using both manual and automatic segmentation procedures. We found a significant difference between the performances of classifiers when manual or automatic segmentation was used. Therefore, an automatic VOI segmentation procedure could affect the overall performance. However, our results proved that, although a single classifier trained separately with dynamic and morphological features achieves a low overall accuracy, the MCS could optimise the correct classification rate: dynamic features with the decision tree gave $60 \%$ accuracy, morphological features

Table 3 Performance obtained by the proposed methods on the testing set

\begin{tabular}{|c|c|c|c|c|c|c|c|}
\hline Classifier & Segmentation & Sensitivity (\%) & Specificity (\%) & PPV (\%) & NPV (\%) & Accuracy (\%) & $p$ value $^{a}$ \\
\hline \multirow{2}{*}{$\begin{array}{l}\text { Bayesian classifier using } \\
\text { dynamic features }\end{array}$} & Manual & $92.3(24 / 26)$ & $81.8(18 / 22)$ & $85.7(24 / 28)$ & $90.0(18 / 20)$ & 87.5 & \multirow[t]{2}{*}{0.04} \\
\hline & Automatic & $88.5(23 / 26)$ & $68.2(15 / 22)$ & $76.7(23 / 30)$ & $83.3(15 / 18)$ & 79.2 & \\
\hline \multirow{2}{*}{$\begin{array}{l}\text { Decision tree classifier using } \\
\text { morphological features }\end{array}$} & Manual & $92.3(24 / 26)$ & $77.3(17 / 22)$ & $82.8(24 / 29)$ & $89.5(17 / 19)$ & 85.4 & \multirow[t]{2}{*}{0.02} \\
\hline & Automatic & $76.9(20 / 26)$ & $40.9(9 / 22)$ & $60.6(20 / 33)$ & $60.0(9 / 15)$ & 60.4 & \\
\hline
\end{tabular}

PPV positive predictive value, NPV negative predictive value 


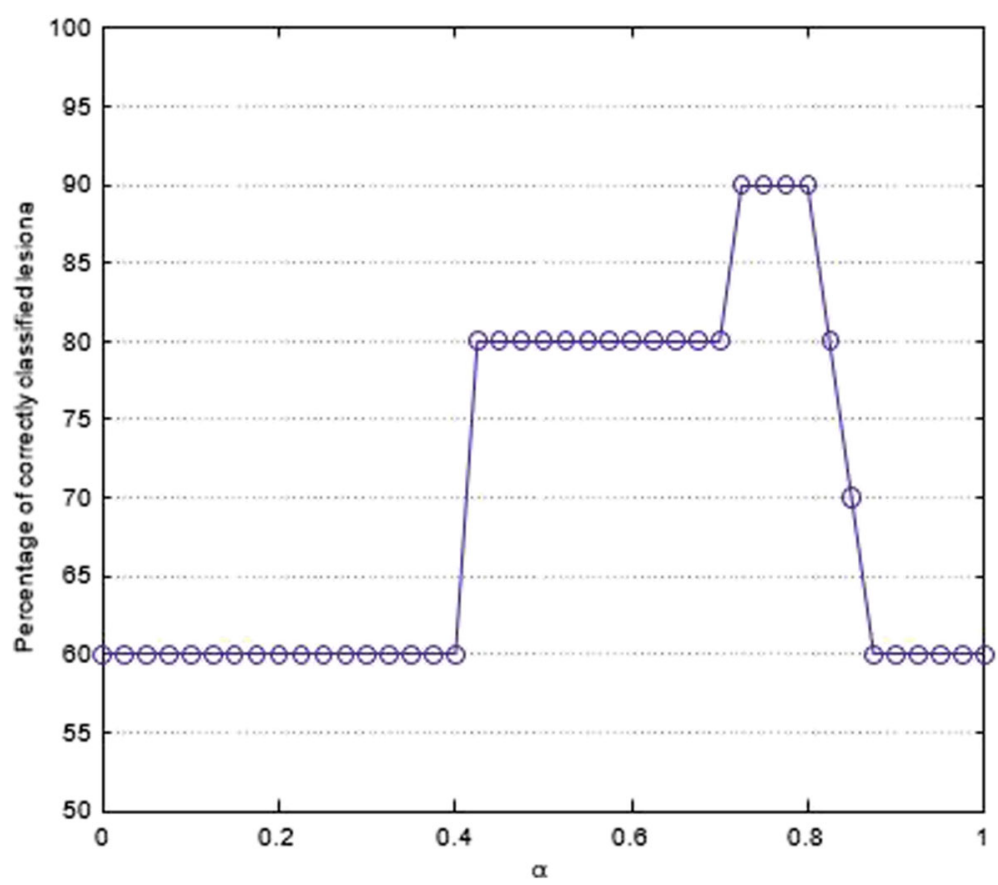

Fig. 3 Percentage of correctly classified lesions by the proposed MCS versus the coefficient $a$

with the Bayes classifier gave $80 \%$ accuracy, and the MCS gave $91.7 \%$ accuracy. Overall accuracy of the MCS is comparable with the accuracy of each of the two classifiers when a manual segmentation procedure was performed. These results are interesting because ROI manual selection performed slice by slice is time consuming and operator dependent.

The findings of this study are in line with recent literature [23, 24]. In fact, Wedegärtner et al. [23] reported a sensitivity of $83 \%$ using morphological features (irregular lesion contour) and an area under the receiver operating characteristic curve of 0.9 for 62 breast lesions without automatic classification. Tzacheva et al. [15] reported a sensitivity of $90 \%$, a specificity of $91 \%$, and an accuracy of $91 \%$ using morphological features and a multilayer perceptron classifier on 14 breast lesions. However, these authors did not use an automatic segmentation step. Zheng et al. [16] reported a sensitivity of $95 \%$ using a combination of temporal, spatial, and morphological attributes and a linear classifier for 31 subjects, but even in this study the segmentation step was not completely automatic. Thus, the novelty of our study is the use of a completely automatic MCS.

Further investigation is required for an optimal choice of $\alpha$ and $\beta$ because the specific value could affect the overall accuracy of the system (see Fig. 3). It is worth noting that there is an interval of values $(0.7,0.8)$ in which high performance can be obtained.

An important limitation of this study is that the classifier was trained and tested only with a small number of subjects. As a consequence, our preliminary results need to be confirmed on a larger number of patients. Moreover, manual segmentation could be done by multiple readers to assess inter-observer variability. Finally, a combination of morphological, dynamic, and also texture features could be performed.

In conclusion, we proposed an MCS to classify breast lesions on DCE-MRI combining the results of two classifiers tested with morphological features and dynamic information separately. The MCS could optimise the correct classification rate, reaching an accuracy of $91.7 \%$.

\section{Authors' contributions}

Each author has participated sufficiently in this submission and takes public responsibility for its content. All authors read and approved the final manuscript.

\section{Competing interests}

The authors declare that they have no competing interests.

Consent for publication

All patients provided informed consent for the use of their data for research purposes.

Ethics approval and consent to participate

This retrospective study was performed according to regulations issued by our local Institutional Review Board.

\section{Publisher's Note}

Springer Nature remains neutral with regard to jurisdictional claims in published maps and institutional affiliations. 


\section{Author details}

"Department of Diagnostic Imaging, Radiant and Metabolic Therapy, "Istituto Nazionale Tumori Fondazione Giovanni Pascale—IRCCS", Via Mariano Semmola, 80131 Naples, Italy. ${ }^{2}$ Department of Melanoma Surgical Oncology, "Istituto Nazionale Tumori Fondazione Giovanni Pascale—IRCCS", Via Mariano Semmola, 80131 Naples, Italy. ${ }^{3}$ Department of Electrical Engineering and Information Technologies, University "Federico II" of Naples, Via Claudio 21, 80125 Naples, Italy.

Received: 11 January 2017 Accepted: 22 March 2017

Published online: 29 June 2017

\section{References}

1. Lehman CD, Gatsonis C, Kuhl CK et al, ACRIN Trial 6667 Investigators Group (2007) MRI evaluation of the contralateral breast in women with recently diagnosed breast cancer. N Engl J Med 356:1295-1303

2. Olsen O, Gøtzsche PC (2001) Screening for breast cancer with mammography. Cochrane Database Syst Rev 4:CD001877

3. Schnall MD, Blume J, Bluemke DA et al (2006) Diagnostic architectural and dynamic features at breast MR imaging: multicenter study. Radiology 238: $42-53$

4. Nie K, Chen JH, Yu HJ et al (2008) Quantitative analysis of lesion morphology and texture features for diagnostic prediction in breast MRI. Acad Radiol 15:1513-1525

5. Liney GP, Sreenivas M, Gibbs P, Garcia-Alvarez R, Turnbull LW (2006) Breast lesion analysis of shape technique: semiautomated vs. manual morphological description. J Magn Reson Imaging 23:493-498

6. Ikeda DM, Hylton NM, Kinkel K et al (2001) Development, standardization, and testing of a lexicon for reporting contrast-enhanced breast magnetic resonance imaging studies. J Magn Reson Imaging 13:889-895

7. Fusco R, Sansone M, Filice $S$ et al (2016) Pattern recognition approaches for breast cancer DCE-MRI classification: a systematic review. J Med Biol Eng 36: 449-459

8. Fusco R, Sansone M, Filice S et al (2015) Integration of DCE-MRI and DWMRI quantitative parameters for breast lesion classification. Biomed Res Int 2015:237863

9. Ikeda DM (2001) Progress report from the American College of Radiology Breast MR Imaging Lexicon Committee. Magn Reson Imaging Clin N Am 9: 295-302

10. Gibbs P, Turnbull LW (2003) Textural analysis of contrast-enhanced MR images of the breast. Magn Reson Med 50:92-98

11. Schlossbauer $T$, Leinsinger $G$, Wismuller $A$ et al (2008) Classification of small contrast enhancing breast lesions in dynamic magnetic resonance imaging using a combination of morphological criteria and dynamic analysis based on unsupervised vector-quantization. Invest Radiol 43:56-64

12. McLaren CE, Chen WP, Nie K, Su MY (2009) Prediction of malignant breast lesions from MRI features: a comparison of artificial neural network and logistic regression techniques. Acad Radiol 16:842-851

13. Zheng Y, Baloch S, Englander S, Schnall MD, Shen D (2007) Segmentation and classification of breast tumor using dynamic contrast-enhanced MR images. Med Image Comput Comput Assist Interv 10:393-40

14. Agner SC, Soman S, Libfeld E et al (2011) Textural kinetics: a novel dynamic contrast-enhanced (DCE)-MRI feature for breast lesion classification. J Digit Imaging 24:446-463

15. Tzacheva AA, Najarian K, Brockway JP (2003) Breast cancer detection in gadolinium-enhanced MR images by static region descriptors and neural networks. J Magn Reson Imaging 17:337-342

16. Zheng Y, Englander S, Baloch S et al (2009) STEP: spatiotemporal enhancement pattern for MR-based breast tumor diagnosis. Med Phys 36: 3192-204

17. Keyvanfard F, Shoorehdeli MA, Teshnehlab M (2013) Specificity enhancement in classification of breast MRI lesion based on multi-classifier. Neural Comput Applic 22:35-45

18. Fusco R, Sansone M, Sansone C, Pepino A, Petrillo A (2012) Classification of breast lesions using dynamic and morphological features in DCE-MRI. In: Proceedings of Congresso Nazionale di Bioingegneria, Rome. 26/06/2012 29/06/2012

19. Fusco R, Sansone M, Sansone C, Petrillo A (2011) Selection of Suspicious ROls in Breast DCE-MRI. In: Image Analysis and Processing- ICIAP 2011. vol. 6978, Ravenna, Italy, pp 48-57.
20. Fusco R, Sansone M, Sansone C, Petrillo A (2012) Segmentation and classification of breast lesions using dynamic features in dynamic contrast enhanced-magnetic resonance imaging. In: Proceedings of 25th IEEE International Symposium on Computer based Medical Systems (CBMS), Rome, Italy

21. Torricelli P, Pecchi A, Luppi G, Romagnoli R (2003) Gadolinium-enhanced MRI with dynamic evaluation in diagnosing the local recurrence of rectal cancer. Abdom Imaging 28:19-27

22. De Santo M, Molinara M, Tortorella F, Vento M (2003) Automatic classification of clustered microcalcifications by a multiple expert system. Pattern Recogn 26:1467-1477

23. Wedegärtner U, Bick U, Wörtler K, Rummeny E, Bongartz G (2001) Differentiation between benign and malignant findings on MR-mammography: usefulness of morphological criteria. Eur Radiol 11:1645-1650

24. Juntu J, Sijbers J, De Backer S, Rajan J, Van Dyck D (2010) Machine learning study of several classifiers trained with texture analysis features to differentiate benign from malignant soft-tissue tumors in T1-MRI images. J Magn Reson Imaging 31:680-689

\section{Submit your manuscript to a SpringerOpen ${ }^{\circ}$ journal and benefit from:}

- Convenient online submission

- Rigorous peer review

- Immediate publication on acceptance

- Open access: articles freely available online

- High visibility within the field

- Retaining the copyright to your article

Submit your next manuscript at $\boldsymbol{\nabla}$ springeropen.com 